\title{
Longitudinal study on Plasmodium falciparum gametocyte carriage following artemether- lumefantrine administration in a cohort of children aged 12-47 months living in Western Kenya, a high transmission area
}

\author{
Ben Andagalu, Joan Mativo, Edwin Kamau ${ }^{*}$ and Bernhards Ogutu
}

\begin{abstract}
Background: The effects that artemether-lumefantrine (AL) has on gametocyte dynamics in the short-term have recently been described. However there is limited long-term longitudinal data on the effect of AL on gametocyte dynamics in asymptomatic children.

Methods: An epidemiological study was conducted in Kombewa, Western Kenya, in which 270 asymptomatic children aged between 12 and 47 months were enrolled. The subjects were randomized to receive either a course of AL or placebo at enrolment. Active follow-up was conducted for one year.

Results: The gametocyte prevalence and density dynamics throughout the study period mirrored that of the asexual forms. The proportion of initially parasitaemic subjects becoming gametocytaemic was significantly lower in the AL arm for the first 12 weeks following randomization. The geometric mean gametocyte density was lower in the AL arm for 2 weeks following randomization. None of the variables of interest had a statistically significant effect on the duration of gametocytaemia. There is no effect seen in subjects who are not parasitaemic at the time of drug administration.
\end{abstract}

Conclusions: The treatment of asymptomatic parasitaemic subjects with AL results in a significant reduction in the proportion of subjects who become gametocytaemic for at least 12 weeks.

Keywords: Gametocytes, Western Kenya, Longitudinal study, Asymptomatic children, Artemether-lumefantrine, Epidemiological study

\section{Background}

Both symptomatic and asymptomatic malaria infected persons tend to harbour gametocytes, the sexual stage of the malaria parasite that mediates its transmission from the infected human host to a susceptible mosquito [1]. Because they are less likely to seek treatment, the asymptomatic carriers constitute a significant reservoir of parasites available for transmission by mosquitoes [2].

\footnotetext{
* Correspondence: edwin.kamau@us.army.mil

Department of Emerging Infectious Diseases-Global Emerging Infections Surveillance and Response System (DEID-GEIS) Program, United States Army Medical Research Unit-Kenya (USAMRU-K), Kenya Medical Research Institute (KEMRI)-Walter Reed Project, P.O. Box 54, 40100 Kisumu, Kenya
}

The proportion of asymptomatic or subclinical Plasmodium falciparum infections in endemic countries is high, with microscopically detected prevalence reported to be as high as $39 \%[3,4]$. A large number of gametocyte carriers have submicroscopic gametocytaemia [4,5]. Studies have shown that molecular methods detect gametocytes 3- to 10-fold more than microscopy [6-8]. Interestingly, even at submicroscopic densities, gametocytes do infect mosquitoes and contribute to malaria transmission $[9,10]$.

Gametocyte carriage in naturally infected individuals in a population is influenced by many factors such as the age group [5,11-13], transmission settings [5,14,15], 
seasons [5,16-18] and the human host genetic factors [19-21]. Other important risk factors for gametocyte carriage include anaemia [22], durations of the infections, recrudescent infections [23], fever [24], mixed infections $[23,25]$ and drug treatment $[26,27]$.

Data on duration of gametocytes carriage in natural infections is limited because such longitudinal studies are challenging to conduct [5]. In a longitudinal study conducted in 1958, volunteers were followed up for one year, with sampling of the parasites done every other day [28]. Eighty percent of the enrolled subjects had detectable gametocytes by microscopy at least once during follow up, with more than half carrying gametocytes 20 times or more, and the longest duration of carriage was estimated at 188 days. In another study, gametocyte carriage was shown to extend to 30 days in Gambian adults in a single transmission season [11]. In a study conducted in western Kenya on asymptomatic children, the mean duration of gametocyte carriage was shown to decrease with increasing age [27]. This was a 30-day longitudinal study. Interestingly, children of $<10$ years could carry gametocytes for three weeks or more.

Treatment of asymptomatic asexual $P$. falciparum carriers directly benefits the carriers given that the clearance of the asexual parasite load prevents anaemia, which in turn is associated with reduced work capacity, poor pregnancy outcomes and reduced cognitive function [2]. Adoption of artemisinin-based combination therapy (ACT) as the first-line treatment of uncomplicated malaria has been beneficial because of their effect on gametocytes and consequently malaria transmission [29-32]. Artemether-lumefantrine (AL) is one of the most widely used ACT in Africa with extensive efficacy and safety data [2]. The effects that AL has on gametocyte dynamics in the short-term have recently been described [2,33]. However, there is limited long-term longitudinal data on the effect that the administration of AL to asymptomatic $P$. falciparum carriers has on gametocyte dynamics. To describe this, data collected from a longitudinal cohort study of the epidemiology of paediatric malaria in Kombewa Division, Western Kenya was analysed.

\section{Methods}

\section{Source of data}

Data was obtained from the longitudinal cohort study of the epidemiology of paediatric malaria in Kombewa Division, Western Kenya. The study was conducted in order to estimate the prevalence of asymptomatic and symptomatic malaria as well as to describe the temporal pattern of episodes of symptomatic malaria among young children living in Kombewa Division. An additional aim was to assess whether administration of a curative dose of an anti-malarial treatment at the beginning of the study leads to a large change in the pattern of development of episodes of malaria during the subsequent follow-up period. The information obtained from this study was for the planning and design of a paediatric malaria vaccine trial that was to be conducted in the same area.

\section{Study area}

Kombewa Division is a 361 square kilometre rural area located near Lake Victoria in the western part of Kenya, with a population of approximately 70,000 and an under-five population of approximately 8,500 . The climate in Kombewa is characterized by two rainy seasons - the long rains (April through June) and the short rains (August through October). The vegetation is largely savanna in nature. Kombewa has year round transmission of malaria, with the most intense transmission occurring during the long rains and the short rains. For the purpose of organizing recruitment and followup of the volunteers, the field site was divided into 22 subdivisions. Within each subdivision in which a participant resided there was a satellite field site that was staffed $24 \mathrm{hrs} /$ day, 7 days/week. Each household participating in the study was thus located within a 1-mile radius of a satellite field site that was accessible during the rainy and dry seasons. The study protocol was reviewed and approved by the Ethical Review Committee of the Kenya Medical Research Institute (KEMRI) and the Human Use Review Committee of the Walter Reed Army Institute of Research (WRAIR) and by the Human Subjects Research and Review Board of the Surgeon General of the US Army at Fort Detrick, Maryland. Parents or guardians of participants were provided written, informed consent before screening and enrolment and had to pass an assessment of understanding.

\section{Study design and procedures}

This was an epidemiological follow-up study, in which a total of 270 initially healthy, asymptomatic children aged between 12 months and 47 months living within the study area were enrolled in three age cohorts of 12-23 months, 24-35 months and 36-47 months. Inclusion criteria included being 12 to 48 months of age, verifiable by appropriate documentation. Subjects with clinical signs of malaria and other significant medical conditions such as homozygous sickle cell disease and malnutrition were excluded. Samples for a baseline malaria blood film (MBF) and a full blood count were collected just before randomization; the results of MBF were not used to determine eligibility, and were not made available to the investigators. The children were then randomized to receive either a course of artemether-lumefantrine (Coartem ${ }^{\circ}$ ) or placebo using a list prepared in advance using a blocked randomization scheme. All study staff with the exception of the study pharmacists and the study coordinator 
were blinded to the allocations. The treatment was prepared by the study pharmacist at the clinic and its administration directly supervised by study team members - the first, third and fifth doses were administered at the clinic, while the second, fourth and sixth doses were administered by field workers at home. Each of the children was then followed up for one year. The follow-up consisted of active surveillance and passive surveillance. Active surveillance consisted of weekly visits by field workers to the participants' homes and scheduled monthly visits by the participants to the study clinic, during which samples for malaria blood films were collected. Haemoglobin determination was done during the monthly visit at the clinic. The results of the MBFs collected during the active surveillance were not availed to the investigators unless the subject was unwell during a scheduled contact. Passive surveillance consisted of unscheduled sick visits by the study participants to the study clinic whenever the participants had specific complaints - during these visits, laboratory assessments including malaria blood films were done whenever the clinical condition warranted. The results of the MBFs taken during sick visits were made available to the investigators. Subjects who were symptomatic and were diagnosed with malaria as per the laboratory assessment during the follow up period were treated with $\mathrm{AL}$, regardless of the randomization group.

\section{Diagnosis of malaria}

Duplicate thick and thin blood smears were made and air dried. Thin blood smear was methanol fixed and both smears were stained with $5 \%$ Giemsa in phosphate buffer for 30 minutes. With high power objective the smears were examined, the number, species and stage of the parasites were recorded. The total numbers of parasites, both asexual and sexual forms per high power field (HPF) were counted against 200 white blood cells. For samples that had high density parasitaemia, the asexual forms were counted against 2,000 red blood cells. A total of $100 \mathrm{HPF}$ were examined before a slide was considered negative, and a minimum of $25 \mathrm{HPF}$ were examined on a positive smear. Two independent microscopists examined the MBFs, and a mean of their findings calculated. Discrepant results were resolved by having a third reader, and the two closest results were taken. The microscopists were proficient and worked under the guidance of malaria diagnostic centre within the programme.

\section{Statistical analysis}

All data were entered and verified in Microsoft Access then imported into STATA software version 10 (StataCorp., College Station, TX). Malaria parasite densities were converted from parasites per 200 white blood cells and parasites per 2,000 red blood cells to parasite $/ \mu \mathrm{L}$, assuming a WBC count of 8000 or a RBC count of
$4 \times 10^{6}$ respectively. The duration of gametocytaemia was calculated by assuming that a single gametocyte positive slide represented gametocytes circulating in the peripheral blood for at least 2.5 days. If participants presented with gametocyte positive MBFs on successive visits, the duration of gametocytaemia was estimated as the interval between the visits plus 2.5 days [34,35]. Covariates of interest were the use of mosquito nets, age, presence of mixed infection (P. falciparum and Plasmodium malariae, or P. falciparum and Plasmodium ovale) and whether or not the child was pre-treated with AL at randomization. The baseline characteristics data is presented of the three age groups; 12-23, 24-35 and 36-47 months in that order respectively. The association between the time to the first or only episode of gametocytaemia, as well as the risk for multiple events of gametocytaemia, and the variables of interest was analysed using Cox regression analysis. The association between continuous outcomes (gametocyte density, gametocytaemia duration) and the outcomes of interest were evaluated using robust regression models.

\section{Results \\ Study profile}

A total of 539 children were screened, 270 met the eligibility criteria and were enrolled into the study where 135 were randomized to receive placebo and 135 randomized to receive AL. Of the 270 volunteers that were enrolled into the study, $69 \%$ (187) completed the study which the follow-up went on for a year. Of those that completed the study, $53 \%$ (99 of 187) were in the placebo arm and $47 \%$ (88 of 187) in the AL arm. Of the 83 volunteers that did not complete the one year follow-up, $81 \%$ (67) withdrew their consent, with 55\% (46 of 83) participants citing their discomfort with the weekly blood draws as the reason for consent withdrawal. Migration out of the study area accounted for the remainder of those who did not complete the study. No deaths were recorded among the study cohort for the duration of the study. However, two serious adverse events (SAEs) were recorded where one child in the AL arm was hospitalized with a diagnosis of severe malaria 19 weeks after randomization - this particular participant had defaulted on clinic visits, while the other child in the placebo arm was hospitalized with severe pneumonia. Both children recovered. All children completed the six doses of the study treatment.

\section{Baseline characteristics}

The baseline characteristics of both treatment arms were generally well balanced (Table 1). Insecticide-treated nets (ITN) use was low in the study cohort (33\% overall) with a slight predominance in the placebo arm (36.3\% versus. 29.6\%). There was a general trend of higher ITN use in the 12-23 months age group than the older age groups (overall $43.3 \%$ versus $30.0 \%$ and $25.6 \%$ ). Parasitological data for the 
Table 1 Baseline characteristics

\begin{tabular}{|c|c|c|c|}
\hline & $A L(N=135)$ & Placebo $(\mathrm{N}=135)$ & Overall (270) \\
\hline Age in months - Mean (SD) ${ }^{a}$ & $29.6(10.2)$ & $30.1(10.1)$ & $29.8(10.1)$ \\
\hline Male - N (\%) & 72/135 (53.3) & $69 / 135(51.1)$ & $141 / 270(52.2)$ \\
\hline $\mathrm{Hb}-$ Mean (SD) & $9.9(1.3)$ & $9.9(1.4)$ & $9.9(1.4)$ \\
\hline Bed net use overall - N (\%) & 40/135 (29.6) & 49/135 (36.3) & $89 / 270(33)$ \\
\hline Bed net use $12-23$ months $-\mathrm{N}(\%)$ & 16/45 (35.6) & $23 / 45(51.1)$ & $39 / 45(43.3)$ \\
\hline Bed net use $24-35$ months $-\mathrm{N}(\%)$ & 15/45 (33.3) & $12 / 45(26.7)$ & $27 / 45(30.0)$ \\
\hline Bed net use $36-47$ months $-\mathrm{N}(\%)$ & $9 / 45(20.0)$ & $14 / 45(31.1)$ & 23/45 (25.6) \\
\hline
\end{tabular}

${ }^{a}$ The standard deviation (SD) from the mean of the sample size analysed.

randomization day was available for 265 subjects. The prevalence of parasitaemia at randomization was expectedly high, considering that enrolment took place over a twoweek period between the end of May and early June, coinciding with the peak of the malaria season (Table 2). A total of $66.4 \%$ (176 of 265) participants had asexual parasitaemia at randomization. The prevalence was lower in bed-net users $(58.1 \%$ versus $70.4 \%)$ and in the youngest age group (52.0\% versus $72.0 \%$ and $76.1 \%)$. Gametocyte prevalence at enrolment was $17.4 \%$, and was higher in bed net users (23.3\% versus $15.0 \%)$ and lower in the youngest age group (16.0\% versus $19.3 \%$ and $17.1 \%$ ). The geometric mean asexual parasite density at enrolment was $3328 \mathrm{p} / \mu \mathrm{L}$ (IQR 940 - 12260) while the geometric mean gametocyte density was $94.1 \mathrm{p} / \mu \mathrm{L}$ (IQR 40 - 120), with little variation between the various groups.

\section{Gametocyte prevalence and risk factors}

The gametocyte prevalence dynamics throughout the study period were similar to the asexual parasite prevalence dynamics, with the gametocyte prevalence peaks coinciding with the asexual parasite prevalence peaks (Figure 1). For subjects who had a positive MBF at enrolment, the proportion of subjects who became gametocytaemic was significantly lower for those who received $\mathrm{AL}$ at randomization $(24.6 \%$ versus. $50.7 \%$, $P=0.002)$. This effect was observed for the first 12 weeks following randomization (Figure 2). For subjects who had a negative malaria smear at enrolment, the difference in the proportion of subjects who became gametocytaemic over the first 12 weeks in the placebo and $\mathrm{AL}$ arms was not statistically significant $(11.6 \%$ versus $5.4 \%$ respectively, $P=0.326$ ). Being in the older age category, AL administration at randomization and bed net use were protective against gametocytaemia, while being in the second age category, having a positive smear at enrolment and hyper-parasitaemia were significantly associated with gametocytaemia (Table $3, P<0.001$ and $P=0.034$ ). A positive malaria smear at enrolment and mixed infection were associated with faster appearance of the first or only episode of gametocytaemia (Table $3, P=0.016$ and $P=0.001)$. A positive smear for malaria at enrolment and anaemia was statistically significantly associated with the development of multiple events of gametocytaemia (Table 3 , both events $P<0.001$ ), while the second age category, hyper-parasitaemia and mixed infection seemed to reduce the risk for multiple events (Table 3, $P=0.01$ and $P=0.007)$.

Table 2 Parasitological data at enrolment

\begin{tabular}{lccccc}
\hline & $\mathbf{N}$ & $\mathbf{N}(\%)^{\mathbf{a}}$ & $\mathbf{p} / \boldsymbol{\mu L},(\mathbf{I Q R})^{\mathbf{b}}$ & $\mathbf{N ~ ( \% )}^{\mathbf{c}}$ & $\mathbf{p} / \mathbf{\mu L},(\mathbf{I Q R})^{\mathbf{d}}$ \\
\hline Overall & 265 & $176 / 265(66.4)$ & $3328(940,12260)$ & $46 / 265(17.4)$ & $94.1(40,120)$ \\
AL & 133 & $92 / 133(69.2)$ & $4016.0(1180,1500)$ & $31 / 133(23.3)$ & $102.0(40,120)$ \\
Placebo & 132 & $84 / 132(64.0)$ & $2710.3(860,10660)$ & $15 / 132(11.4)$ & $80.0(40,120)$ \\
Bed net users & 86 & $50 / 86(58.1)$ & $2916.0(880,10440)$ & $20 / 86(23.3)$ & $112.0(40,140)$ \\
Bed net non users & 179 & $126 / 179(70.4)$ & $3509.0(960,13080)$ & $26 / 179(15.0)$ & $83.0(40,120)$ \\
Age 12-23 mo & 89 & $46 / 89(52.0)$ & $3296.0(840,14560)$ & $14 / 89(16)$ & $100.0(40,120)$ \\
Age 24-35 mo & 88 & $63 / 88(72.0)$ & $3067.0(720,10480)$ & $17 / 88(19.3)$ & $101.0(40,120)$ \\
Age 36-47 mo & 88 & $67 / 88(76.1)$ & $3620.3(1440,12480)$ & $15 / 88(17.1)$ & $75.2(40,120)$ \\
Anemic & 60 & $42 / 60(70)$ & $4373.3(1760,13360)$ & $8 / 60(13.3)$ & $115.4(40,100)$ \\
Not anemic & 194 & $128 / 194(66.0)$ & $3117.0(780,12720)$ & $37 / 194(19.1)$ & $92.2(40,120)$ \\
\hline
\end{tabular}

${ }^{\mathrm{a} A s e x u a l}$ parasite prevalence.

${ }^{\mathrm{b}}$ Asexual parasite density $(\mathrm{p} / \mu \mathrm{L})$, Geometric mean (Inter Quartile Range [IQR]).

${ }^{\mathrm{C}}$ Gametocyte prevalence N (\%).

${ }^{\mathrm{d} G a m e t o c y t e ~ d e n s i t y ~(p / \mu L), ~ G e o m e t r i c ~ m e a n ~(I n t e r ~ Q u a r t i l e ~ R a n g e ~[I Q R]) . ~}$ 


\section{Parasite prevalence}

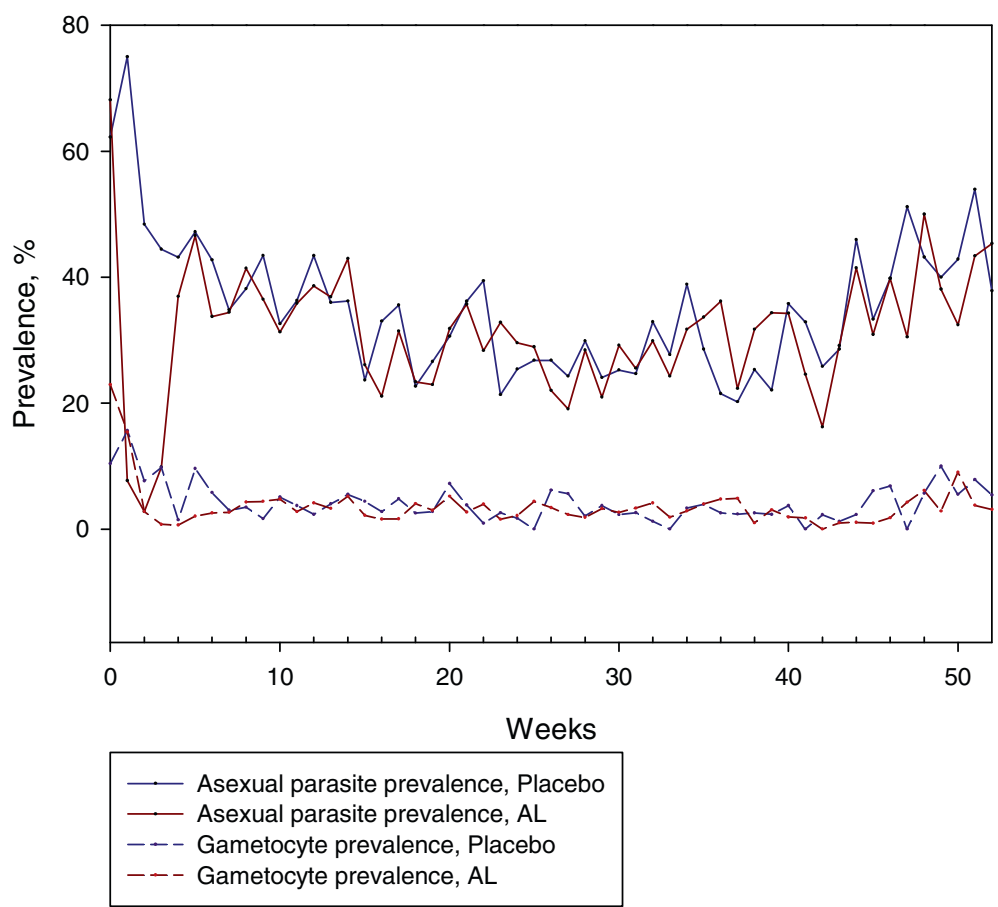

Figure 1 Gametocyte prevalence dynamics. Data showing the percent prevalence of asexual and sexual parasites throughout the study period. The highest prevalence of asexual and sexual parasites was $66.4 \%$ and $17.4 \%$ respectively which was at the beginning of the study. This coincided with height of malaria transmission season. The prevalence of sexual parasite mirrored asexual parasite throughout the study period.

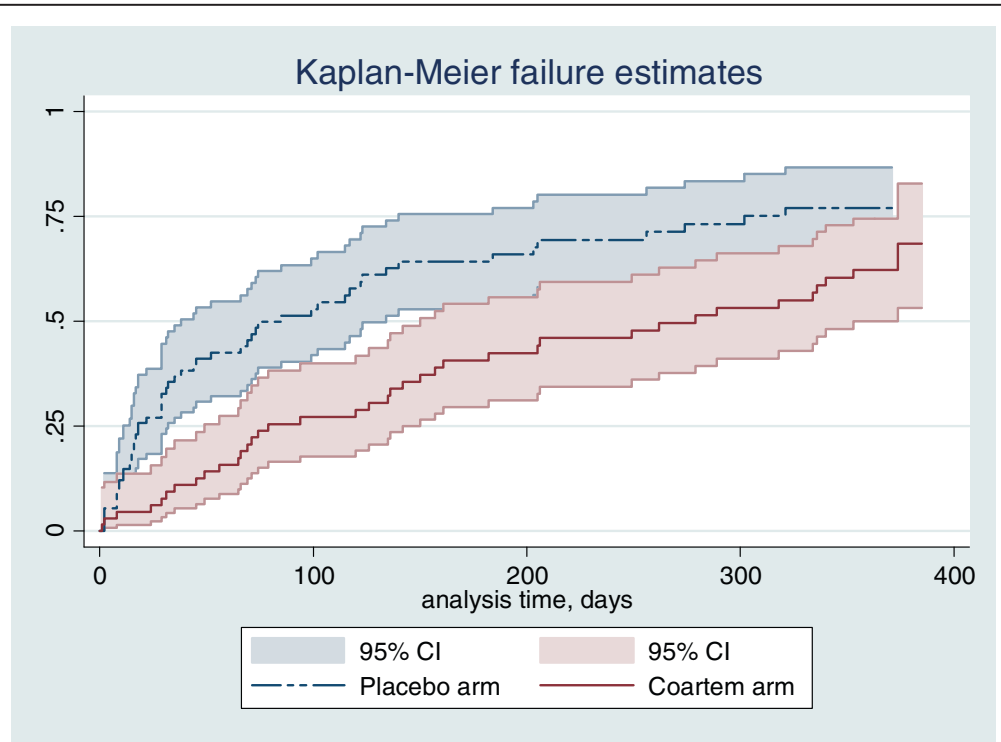

Figure 2 Kaplan-Meier failure estimates showing the proportion of initially parasitaemic participants becoming gametocytaemic. Participant who received AL at randomization had significantly lower gametocytes compared to placebo for the first 12 weeks. 
Table 3 Gametocyte risk factors

\begin{tabular}{cccc}
\hline Variables & $\begin{array}{c}\text { Gametocytaemia versus variables } \\
\text { OR }(\mathbf{9 5} \% \mathbf{C l}) \mathbf{p} \text {-value }\end{array}$ & $\begin{array}{c}\text { First event versus variables } \\
\text { HR }(\mathbf{9 5 \%} \mathbf{C l}) \mathbf{p} \text {-value }\end{array}$ & $\begin{array}{c}\text { Multiple events versus variables } \\
\text { HR }(\mathbf{9 5} \% \mathbf{C l}) \mathbf{p} \text {-value }\end{array}$ \\
\hline Age category 2 & $1.05(1.00,1.11) 0.036$ & $0.44(0.16,1.23) 0.118$ & $0.63(0.44,0.89) 0.01$ \\
Age category 3 & $0.36(0.17,0.74) 0.005$ & $0.75(0.29,1.90) 0.54$ & $0.76(0.54,0.89) 0.01$ \\
Bed net use & $0.26(0.08,0.91) 0.035$ & $1.32(0.57,3.05) 0.515$ & $1.34(1.01,1.80) 0.46$ \\
Pf enrolment & $4.46(2.94,6.74)<0.001$ & $3.36(1.26,8.98) 0.016$ & $3.56(2.37,5.37)<0.001$ \\
Anaemia & $1.24(0.82,1.88) 0.299$ & $1.46(0.55,3.87) 0.447$ & $2.06(1.49,2.85)<0.001$ \\
Hyper Pf & $1.44(1.03,2.01) 0.034$ & $0.86(0.20,3.78) 0.845$ & $0.34(0.16,0.74) 0.007$ \\
Pretreatment & $1.81(1.20,2.72) 0.004$ & $0.85(0.38,1.88) 0.682$ & $0.81(0.62,1.06) 0.128$ \\
Mixed Infection & $1.05(1.00,1.11) 0.036$ & $4.89(1.99,11.99) 0.001$ & $0.63(0.44,0.89) 0.01$ \\
\hline
\end{tabular}

${ }^{a}$ Plasmodium falciparum positive participants at enrolment.

${ }^{b}$ Participants with hyper parasitaemia which is $100,000 \mathrm{p} / \mathrm{mL}$ and above.

Odds ratio (OR), hazardous ratio (HR), the $95 \%$ confidence interval $(95 \% \mathrm{Cl})$ and p-values based on Cox regression analysis were generated. Association between gametocytaemia, the time to the first or only episode of gametocytaemia, the risk for multiple events of gametocytaemia, with the variables of interest were determined.

\section{Gametocyte density and duration}

The gametocyte density dynamics were similar to the asexual parasite density dynamics, with the peaks coinciding with the asexual parasite density peaks (Figure 3 ). The geometric mean density of asexual as well as sexual forms of the parasite was lower in the $\mathrm{AL}$ arm for 2 weeks following randomization. However, the densities actually surpassed those in the placebo arm after the third week before they returned to levels similar to those in the placebo arm (Figure 4). Gametocyte density was positively associated with asexual parasite density (Table 4, $P=0.008)$ and negatively associated with haemoglobin levels (Table $4, P<0.001$ ). The mean gametocytaemia duration was 7.4 days (range $2.5-41.5$ ) in the lowest age category, 5.6 days (range $2.5-37.5$ ) in the middle age category and 6.2 days (range 2.5 - 44.5) in the oldest age category. There was no statistically significant difference in the gametocytaemia duration between the age categories groups as determined by one-way ANOVA $(\mathrm{F}(2,443)=2.11, P=0.12)$.

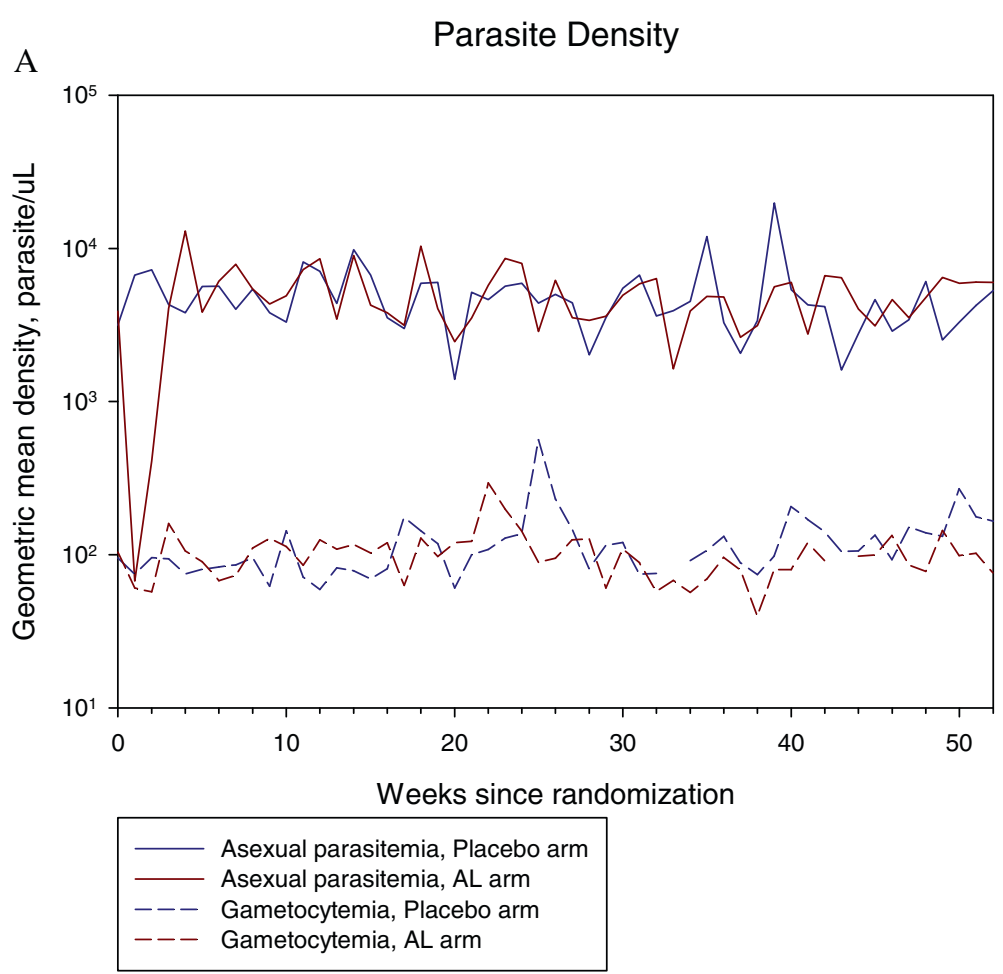

Figure 3 The dynamics of sexual and asexual parasite density: shows the dynamic of asexual and sexual parasite dynamics of both AL and placebo arms throughout the study. 

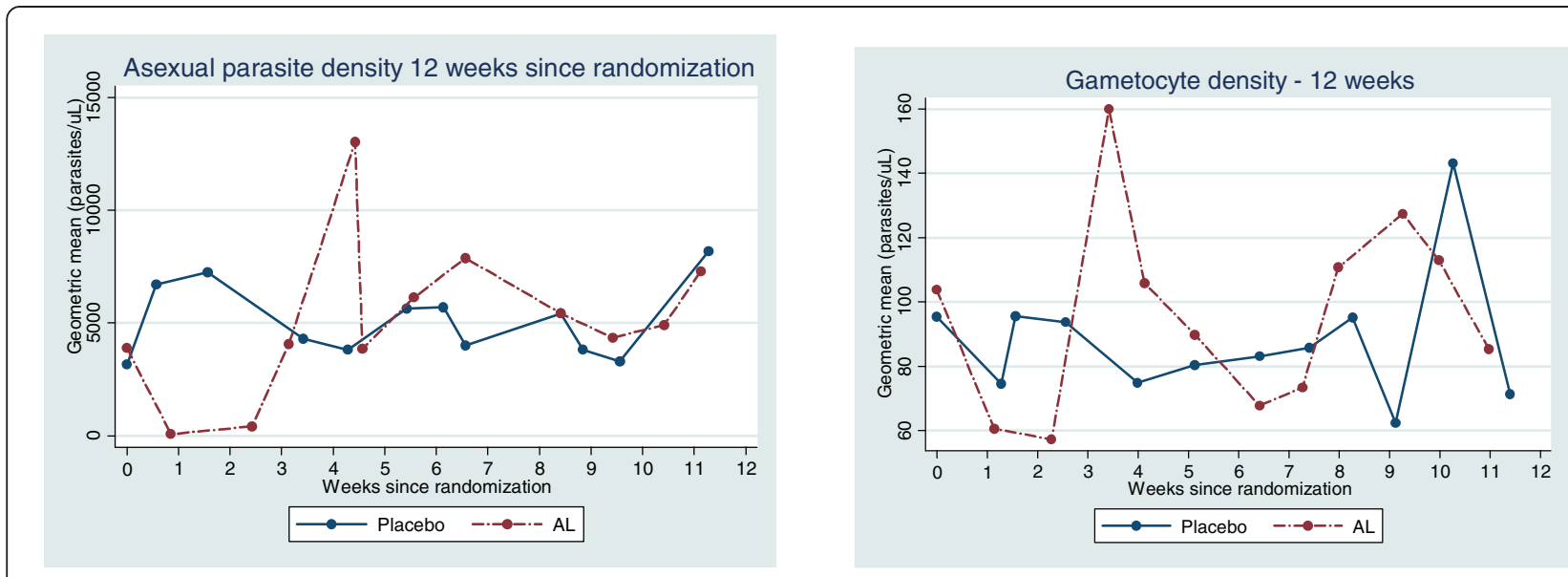

Figure 4 The dynamics of sexual and asexual parasite density: shows the dynamics of asexual and sexual parasite dynamics of both $\mathrm{AL}$ and placebo arms in the first 12 weeks of randomization.

The duration was similar in those who had mixed infection versus those who did not (mean duration of 6.6 versus 6.3 days respectively, $P=0.8$ ). None of the variables of interest had a statistically significant effect on the duration of gametocytaemia in the regression model.

\section{Discussion}

This study presents longitudinal data on gametocytes carriage in natural infections in a cohort of children aged between 12 and 47 months old who were followed for one year. The data shows that treatment of asymptomatic parasitaemic subjects with AL results in a significant reduction in the proportion of subjects who become gametocytaemic for at least 12 weeks, despite AL having a relatively short half-life. The half-life of artemether is estimated to be $3.9 \mathrm{hrs}$ and that of lumefantrine, the longer acting partner is estimated to be

Table 4 Gametocyte density analysis

\begin{tabular}{|c|c|c|}
\hline \multicolumn{2}{|r|}{ Coef $(95 \% \mathrm{Cl})$} & $P$ value \\
\hline Age & $-0.327(-0.840,0.185)$ & 0.211 \\
\hline Parasitaemia & 0.0000364 (9.33E-06, 6.36E-05) & 0.008 \\
\hline $\mathrm{Hb}$ & $-8.009(-12.355,-3.66334)$ & $<0.001$ \\
\hline Mixed Infection & $-4.167(-21.569,13.236)$ & 0.639 \\
\hline Age & $-0.388(-0.896,0.121)$ & 0.135 \\
\hline Parasitaemia & 3.29E-05 (6.65E-06, 5.91E-05) & 0.014 \\
\hline $\mathrm{Hb}$ & $-7.995(-12.389,-3.602)$ & $<0.001$ \\
\hline Body Temp & $-0.874(-4.399,2.651)$ & 0.627 \\
\hline Mixed Infection & $-2.480(-20.443,15.484)$ & 0.787 \\
\hline $\begin{array}{l}\text { Regression coeffic } \\
\text { based on robust } r \\
\text { association betwe } \\
\text { outcomes of seve } \\
\text { which has been } \mathrm{s} \\
\text { the analysis. }\end{array}$ & $\begin{array}{l}\text { oef), the } 95 \% \text { confidence interval }(95 \% \mathrm{Cl}) \text { an } \\
\text { on model were generated. This analysis was } \\
\text { netocyte density, which is a continuous outc } \\
\text { ables as shown. Variables with and without b } \\
\text { ed to affect gametocyte density were separa }\end{array}$ & $\begin{array}{l}\text { alues } \\
\text { to asses } \\
\text { and the } \\
\text { emp, }\end{array}$ \\
\hline
\end{tabular}

$32.7 \mathrm{hrs}$ [36]. There was no effect seen in subjects who were not parasitaemic at enrolment. Studies comparing the risk of gametocyte carriage after treatment with $\mathrm{AL}$ versus dihydroartemisinin-piperaquine (DP) have had mixed results, with some studies showing no difference $[37,38]$, some showing increased risk after treatment with AL [39,40] and DP [1,41-43]. Such differences might be accounted for by the detection method used in these studies and/or transmission intensity. Important also might be whether the study subjects were parasitaemic or not at the time of drug administration, which data here shows that this indeed matters. In a recent study, Sawa et al., showed that AL had a more pronounced effect on malaria transmission shortly after treatment compared to DP [44]. This was the first study to have directly determined gametocyte transmission to mosquitoes that fed on post-treatment blood samples. These authors speculated that AL may be the most appropriate first-line choice for reducing communitywide transmission of $P$. falciparum in setting of low endemicity. AL may be also a good candidate drug for mass screening and treatment of malaria as well as Focal screen and treat in the setting of high endemicity.

In line with other studies, the gametocyte prevalence dynamics throughout the study period followed closely that of the asexual parasite [11,27]. The maximum gametocytaemia duration has been estimated at 22.2 days [40] using microscopy and 107.7 days [41] using molecular methods, which was after non-ACT treatment of the subjects. The maximum gametocytaemia duration in this study was 44.5 days. It is plausible that parasite density was submicroscopic on days when it was recorded as zero and much longer gametocytes durations could have been estimated using molecular methods. This study showed no evidence of higher gametocyte density in the low malaria season as had been suggested before [1]. In areas of high malaria 
transmission intensity, gametocyte carriage is most prevalent in younger age groups, usually grouped as $<5$ years old $[11,27]$. In this study, there were no variations of gametocyte prevalence between the age categories, further supporting clustering of $<5$ years age group together in gametocyte prevalence analysis.

Having parasitaemia at enrolment and mixed infection had a statistically significant influence on the time to the occurrence of the first or only episode of gametocytaemia. These findings are in line with what previous studies have shown that mixed infection increases $P$. falciparum gametocyte production $[29,45]$. Unlike the previous studies that looked at co-infection with $P$. malariae only, the current study looked at co-infection with both $P$. malariae and/or $P$. ovale. Interestingly, being hyper parasitaemic or anaemic did not have a statistically significant influence on the time to the occurrence of the first or only event of gametocytaemia. Anaemia is considered to be a risk factor for gametocytaemia [2]. Although many factors have been speculated to be important in this relationship, cross-species immune responses seem to be the most plausible because hyperparasitaemia or anaemia did not play a critical role. The study data presented here support a phenomenon where mixed infections trigger P. falciparum gametogenesis and hence increased transmission potential. Mixed infections therefore play an important role in prevalence and transmission of falciparum malaria.

This study had three main limitations: First, while the under-five age group was looked at exclusively, an important age category of this group - that of children aged below 1 year - was not looked at. The contribution of this age category in malaria transmission remains largely unknown. Second, there is lack of parasite molecular data that would have complemented the findings of this study. This is in light of the knowledge that sub-microscopic gametocytaemia plays a key role in malaria transmission, and that 3-10 fold increase in gametocyte carriage as determined by microscopy is seen when molecular detection methods are used [4,6-8,46]. Lastly, the original study was designed to address a different research question, and this may affect the statistical power of this study.

\section{Conclusion}

This study provides key insights on the effect that treatment of asymptomatic carriers on malaria transmission. The treatment of asymptomatic parasitaemic subjects with AL results in a significant reduction in the proportion of subjects who become gametocytaemic for at least 12 weeks. There is no effect seen in subjects who are not parasitaemic at the time of drug administration.

\section{Competing interests}

The authors declare that they have no competing interests.

\section{Authors' contributions}

$\mathrm{BO}$ was the study PI. BA, EK and BO conceived and executed the study (data analysis). BA and JM analysed the data. BA, JM, EK wrote the manuscript. BO reviewed the manuscript. All authors read and approved the final manuscript.

\section{Acknowledgements}

We would like to thank the study participants and their parents, the study team in Kombewa, COL Gray Heppner (WRAIR) and Dr. Carter Diggs (USAID) for their participation and support that they provided to this study. The opinions and assertions contained herein are the private opinions of the authors and are not to be construed as reflecting the views of the US Army Medical Research Unit-Kenya or the US Department of Defense.

\section{Funding}

This study was supported by the USAID and Global Emerging Infections Surveillance and Response System (GEIS), a division at the Armed Forces Health Surveillance Center (AFHSC). The funders had no role in study design, data collection and analysis decision to publish, or preparation of the manuscript.

Received: 17 January 2014 Accepted: 5 July 2014

Published: 9 July 2014

\section{References}

1. Bousema T, Drakeley C: Epidemiology and infectivity of Plasmodium falciparum and Plasmodium vivax gametocytes in relation to malaria control and elimination. Clin Microbiol Rev 2011, 24:377-410.

2. Ogutu B, Tiono AB, Makanga M, Premji Z, Gbadoe AD, Ubben D, Marrast AC, Gaye O: Treatment of asymptomatic carriers with artemetherlumefantrine: an opportunity to reduce the burden of malaria? Malar J 2010, 9:30

3. Mabunda S, Aponte JJ, Tiago A, Alonso P: A country-wide malaria survey in Mozambique. II. Malaria attributable proportion of fever and establishment of malaria case definition in children across different epidemiological settings. Malar J 2009, 8:74

4. Tiono A, Ouedraogo A, Ogutu B, Diarra A, Coulibaly S, Gansane A, Sirima S, O'Neil G, Mukhopadhyay A, Hamed K: A controlled, parallel, cluster-randomized trial of community-wide screening and treatment of asymptomatic carriers of Plasmodium falciparum in Burkina Faso. Malar J 2013, 12:79.

5. Drakeley C, Sutherland C, Bousema JT, Sauerwein RW, Targett GA The epidemiology of Plasmodium falciparum gametocytes: weapons of mass dispersion. Trends Parasitol 2006, 22:424-430.

6. Mens PF, Sawa P, van Amsterdam SM, Versteeg I, Omar SA, Schallig HD, Kager PA: A randomized trial to monitor the efficacy and effectiveness by QT-NASBA of artemether-lumefantrine versus dihydroartemisininpiperaquine for treatment and transmission control of uncomplicated Plasmodium falciparum malaria in western Kenya. Malar J 2008, 7:237.

7. Schneider P, Bousema T, Omar S, Gouagna L, Sawa P, Schallig H, Sauerwein R: (Sub)microscopic Plasmodium falciparum gametocytaemia in Kenyan children after treatment with sulphadoxine-pyrimethamine monotherapy or in combination with artesunate. Int J Parasitol 2006, 36:403-408.

8. Schneider P, Schoone G, Schallig H, Verhage D, Telgt D, Eling W, Sauerwein $\mathrm{R}$ : Quantification of Plasmodium falciparum gametocytes in differential stages of development by quantitative nucleic acid sequence-based amplification. Mol Biochem Parasitol 2004, 137:35-41.

9. Ouedraogo AL, Bousema T, Schneider P, de Vlas SJ, Ilboudo-Sanogo E, Cuzin-Ouattara N, Nebie I, Roeffen W, Verhave JP, Luty AJ, Sauerwein R: Substantial contribution of submicroscopical Plasmodium falciparum gametocyte carriage to the infectious reservoir in an area of seasonal transmission. PLoS One 2009, 4:e8410.

10. Schneider P, Bousema JT, Gouagna LC, Otieno S, van de Vegte-Bolmer M, Omar SA, Sauerwein RW: Submicroscopic Plasmodium falciparum gametocyte densities frequently result in mosquito infection. Am J Trop Med Hyg 2007, 76:470-474.

11. Drakeley CJ, Akim NI, Sauerwein RW, Greenwood BM, Targett GA: Estimates of the infectious reservoir of Plasmodium falciparum malaria in The Gambia and in Tanzania. Trans R Soc Trop Med Hyg 2000, 94:472-476.

12. Githeko AK, Brandling-Bennett AD, Beier M, Atieli F, Owaga M, Collins FH: The reservoir of Plasmodium falciparum malaria in a holoendemic area of western Kenya. Trans R Soc Trop Med Hyg 1992, 86:355-358. 
13. Mabunda S, Casimiro S, Quinto L, Alonso P: A country-wide malaria survey in Mozambique. I. Plasmodium falciparum infection in children in different epidemiological settings. Malar J 2008, 7:216.

14. Drakeley CJ, Carneiro I, Reyburn H, Malima R, Lusingu JP, Cox J, Theander TG, Nkya WM, Lemnge MM, Riley EM: Altitude-dependent and -independent variations in Plasmodium falciparum prevalence in northeastern Tanzania. J Infect Dis 2005, 191:1589-1598.

15. Mwangi TW, Ross A, Snow RW, Marsh K: Case definitions of clinical malaria under different transmission conditions in Kilifi District, Kenya. J Infect Dis 2005, 191:1932-1939.

16. Boudin C, Robert V, Verhave JP, Carnevale P, Ambroise-Thomas P: Plasmodium falciparum and P. malariae epidemiology in a West African village. Bull World Health Organ 1991, 69:199-205.

17. Oesterholt MJ, Bousema JT, Mwerinde OK, Harris C, Lushino P, Masokoto A Mwerinde $\mathrm{H}$, Mosha FW, Drakeley CJ: Spatial and temporal variation in malaria transmission in a low endemicity area in northern Tanzania. Malar J 2006, 5:98

18. Ouedraogo AL, de Vlas SJ, Nebie I, Ilboudo-Sanogo E, Bousema JT, Ouattara AS, Verhave JP, Cuzin-Ouattara N, Sauerwein RW: Seasonal patterns of Plasmodium falciparum gametocyte prevalence and density in a rural population of Burkina Faso. Acta Trop 2008, 105:28-34.

19. Gouagna LC, Bancone G, Yao F, Yameogo B, Dabire KR, Costantini C, Simpore J, Ouedraogo JB, Modiano D: Genetic variation in human HBB is associated with Plasmodium falciparum transmission. Nat Genet 2010, 42:328-331

20. Lawaly YR, Sakuntabhai A, Marrama L, Konate L, Phimpraphi W, Sokhna C, Tall A, Sarr FD, Peerapittayamongkol C, Louicharoen C, Schneider BS, Levescot A, Talman A, Casademont I, Menard D, Trape JF, Rogier C, Kaewkunwal J, Sura T, Nuchprayoon I, Ariey F, Baril L, Singhasivanon P, Mercereau-Puijalon O, Paul R: Heritability of the human infectious reservoir of malaria parasites. PLoS One 2010, 5:e11358.

21. Paganotti GM, Palladino C, Modiano D, Sirima BS, Raberg L, Diarra A, Konate A, Coluzzi M, Walliker D, Babiker HA: Genetic complexity and gametocyte production of Plasmodium falciparum in Fulani and Mossi communities in Burkina Faso. Parasitology 2006, 132:607-614.

22. von Seidlein $L$, Bojang $K$, Jones $P$, Jaffar $S$, Pinder M, Obaro S, Doherty $T$, Haywood M, Snounou G, Gemperli B, Gathmann I, Royce C, McAdam K, Greenwood B: A randomized controlled trial of artemether/benflumetol, a new antimalarial and pyrimethamine/sulfadoxine in the treatment of uncomplicated falciparum malaria in African children. Am J Trop Med Hyg 1998, 58:638-644

23. Price $R$, Nosten F, Simpson JA, Luxemburger C, Phaipun $L$, ter Kuile F, van Vugt $M$, Chongsuphajaisiddhi T, White NJ: Risk factors for gametocyte carriage in uncomplicated falciparum malaria. Am J Trop Med Hyg 1999, 60:1019-1023.

24. Stepniewska K, Price RN, Sutherland CJ, Drakeley CJ, von Seidlein L, Nosten F, White NJ: Plasmodium falciparum gametocyte dynamics in areas of different malaria endemicity. Malar J 2008, 7:249.

25. Bousema JT, Drakeley CJ, Mens PF, Arens T, Houben R, Omar SA, Gouagna LC, Schallig H, Sauerwein RW: Increased Plasmodium falciparum gametocyte production in mixed infections with P. malariae. Am J Trop Med Hyg 2008, 78:442-448.

26. Beavogui AH, Djimde AA, Gregson A, Toure AM, Dao A, Coulibaly B, Ouologuem D, Fofana B, Sacko A, Tekete M, Kone A, Niare O, Wele M, Plowe CV, Picot S, Doumbo OK: Low infectivity of Plasmodium falciparum gametocytes to Anopheles gambiae following treatment with sulfadoxine-pyrimethamine in Mali. Int J Parasitol 2010, 40:1213-1220.

27. Bousema JT, Gouagna LC, Drakeley CJ, Meutstege AM, Okech BA, Akim IN, Beier JC, Githure JI, Sauerwein RW: Plasmodium falciparum gametocyte carriage in asymptomatic children in western Kenya. Malar J 2004, 3:18

28. Miller MJ: Observations on the natural history of malaria in the semiresistant West African. Trans R Soc Trop Med Hyg 1958, 52:152-168.

29. Barnes Kl, Chanda P, Ab Barnabas G: Impact of the large-scale deployment of artemether/lumefantrine on the malaria disease burden in Africa: case studies of South Africa, Zambia and Ethiopia. Malar J 2009, 12:S8.

30. Barnes KI, Durrheim DN, Little F, Jackson A, Mehta U, Allen E, Dlamini SS, Tsoka J, Bredenkamp B, Mthembu DJ, White NJ, Sharp BL: Effect of artemether-lumefantrine policy and improved vector control on malaria burden in KwaZulu-Natal South Africa. PLoS Med 2005, 2:e330.

31. Bhattarai A, Ali AS, Kachur SP, Martensson A, Abbas AK, Khatib R, Al-Mafazy AW, Ramsan M, Rotllant G, Gerstenmaier JF, Molteni F, Abdulla S,
Montgomery SM, Kaneko A, Björkman A: Impact of artemisinin-based combination therapy and insecticide-treated nets on malaria burden in Zanzibar. PLoS Med 2007, 4:e309.

32. Price RN, Nosten F, Luxemburger C, ter Kuile FO, Paiphun L, Chongsuphajaisiddhi T, White NJ: Effects of artemisinin derivatives on malaria transmissibility. Lancet 1996, 347:1654-1658.

33. Kakuru A, Jagannathan P, Arinaitwe E, Wanzira H, Muhindo M, Bigira V, Osilo E, Homsy J, Kamya MR, Tappero JW, Dorsey G: The effects of ACT treatment and TS prophylaxis on Plasmodium falciparum gametocytemia in a cohort of young Ugandan children. Am J Trop Med Hyg 2013, 88:736-743.

34. Drakeley CJ, Flobbe K, Greenwood BM, Targett GA: Plasmodium falciparum gametocytes in Gambian adults. Ann Trop Med Parasitol 2000, 94:399-401.

35. Smalley ME, Sinden RE: Plasmodium falciparum gametocytes: their longevity and infectivity. Parasitology 1977, 74:1-8

36. Mwesigwa J, Parikh S, McGee B, German P, Drysdale T, Kalyango JN, Clark TD, Dorsey G, Lindegardh N, Annerberg A, Rosenthal PJ, Kamya MR, Aweeka F: Pharmacokinetics of artemether-lumefantrine and artesunateamodiaquine in children in Kampala, Uganda. Antimicrob Agents Chemother 2009, 54:52-59.

37. Arinaitwe E, Sandison TG, Wanzira H, Kakuru A, Homsy J, Kalamya J, Kamya MR, Vora N, Greenhouse B, Rosenthal PJ, Tappero J, Dorsey G: Artemether-lumefantrine versus dihydroartemisinin-piperaquine for falciparum malaria: a longitudinal, randomized trial in young Ugandan children. Clin Infect Dis 2009, 49:1629-1637.

38. Zongo I, Dorsey G, Rouamba N, Dokomajilar C, Sere Y, Rosenthal PJ, Ouedraogo JB: Randomized comparison of amodiaquine plus sulfadoxine-pyrimethamine, artemether-lumefantrine, and dihydroartemisinin-piperaquine for the treatment of uncomplicated Plasmodium falciparum malaria in Burkina Faso. Clin Infect Dis 2007, 45:1453-1461.

39. Kamya MR, Yeka A, Bukirwa H, Lugemwa M, Rwakimari JB, Staedke SG, Talisuna AO, Greenhouse B, Nosten F, Rosenthal PJ, Wabwire-Mangen F, Dorsey $\mathrm{G}$ : Artemether-lumefantrine versus dihydroartemisinin-piperaquine for treatment of malaria: a randomized trial. PLOS Clin Trials 2007, 2:e20.

40. Yeka A, Dorsey G, Kamya MR, Talisuna A, Lugemwa M, Rwakimari JB, Staedke SG, Rosenthal PJ, Wabwire-Mangen F, Bukirwa H: Artemether-lumefantrine versus dihydroartemisinin-piperaquine for treating uncomplicated malaria: a randomized trial to guide policy in Uganda. PLoS One 2008, 3:e2390.

41. Tiburcio M, Silvestrini F, Bertuccini L, Sander AF, Turner L, Lavstsen T, Alano P: Early gametocytes of the malaria parasite Plasmodium falciparum specifically remodel the adhesive properties of infected erythrocyte surface. Cell Microbio/ 2012 http://dx.doi.org/10.1111/cmi.12062.

42. Bassat $Q$, Mulenga M, Tinto H, Piola P, Borrmann S, Menendez C, Nambozi M, Valea I, Nabasumba C, Sasi P, Bacchieri A, Corsi M, Ubben D, Talisuna A, D'Alessandro U: Dihydroartemisinin-piperaquine and artemether-lumefantrine for treating uncomplicated malaria in African children: a randomised, non-inferiority trial. PLoS One 2009, 4:e7871.

43. Nambozi M, Van Geertruyden JP, Hachizovu S, Chaponda M, Mukwamataba D, Mulenga M, Ubben D, D'Alessandro U: Safety and efficacy of dihydroartemisinin-piperaquine versus artemetherlumefantrine in the treatment of uncomplicated Plasmodium falciparum malaria in Zambian children. Malar J 2011, 10:50.

44. Sawa P, Shekalaghe SA, Drakeley CJ, Sutherland CJ, Mweresa CK, Baidjoe AY, Manjurano A, Kavishe RA, Beshir KB, Yussuf RU, Omar SA, Hermsen CC, Okell $L$, Schallig HD, Sauerwein RW, Hallett RL, Bousema T: Malaria transmission after artemether-lumefantrine and dihydroartemisinin-piperaquine: a randomized trial. J Infect Dis 2013, 207:1637-1645.

45. Ratcliff A, Siswantoro H, Kenangalem E, Maristela R, Wuwung RM, Laihad F, Ebsworth EP, Anstey NM, Tjitra E, Price RN: Two fixed-dose artemisinin combinations for drug-resistant falciparum and vivax malaria in Papua, Indonesia: an open-label randomised comparison. Lancet 2007, 369:757-765.

46. Bousema JT, Schneider P, Gouagna LC, Drakeley CJ, Tostmann A, Houben R, Githure JI, Ord R, Sutherland C, Omar SA, Sauerwein RW: Moderate effect of artemisinin-based combination therapy on transmission of Plasmodium falciparum. J Infect Dis 2006, 193:1151-1159.

\section{doi:10.1186/1475-2875-13-265}

Cite this article as: Andagalu et al:: Longitudinal study on Plasmodium falciparum gametocyte carriage following artemether-lumefantrine administration in a cohort of children aged 12-47 months living in Western Kenya, a high transmission area. Malaria Journal 2014 13:265. 